\title{
STRONGLY SPLITTING WEIGHTED SHIFT OPERATORS ON BANACH SPACES AND UNICELLULARITY
}

\author{
M. T. KARAEV AND M. GÜRDAL
}

\begin{abstract}
We introduce the notion of strong splitting operator on a separable Banach space, and prove a structure theorem for this operator. We consider the weighted shift operator $T$, $T e_{n}=\lambda_{n} e_{n+1}, n \geqslant 0$, acting in the Banach space $X$ with basis $\left\{e_{n}\right\}_{n \geqslant 0}$. We give some sufficient conditions for $X$ and for the weight sequence $\left\{\lambda_{n}\right\}_{n \geqslant 0}$ under which the operator is unicellular, that is, every nontrivial invariant subspace $E$ of $T$ has the form $E=X_{i}:=\operatorname{Span}\left\{e_{k}: k \geqslant i\right\}$ for some $i \geqslant 1$; and prove that the restricted operators $T \mid X_{i} \quad(i \geqslant 1)$ are strong splitting. Moreover, we describe in terms of so-called discrete Duhamel operator and diagonal operator all extended eigenvectors of the operators $T \mid X_{i}(i \geqslant 1)$.
\end{abstract}

Mathematics subject classification (2010): Primary 47B38, 47A15; Secondary 46B15.

Keywords and phrases: Banach space, weighted shift operator, strong splitting operator, invariant subspace, Duhamel product.

\section{REFERENCES}

[1] N. K. NikOLSKI, Treatise on the shift operator, vol. 273, Springer, Berlin, 1986.

[2] M. T. KARAEV, On extended eigenvalues and extended eigenvectors of some operator classes, Proc. Amer. Math. Soc., 134 (2006), 2383-2392.

[3] N. K. NikOLSKI, Basisness and unicellularity of weighted shift operators, Izvestiya Akad. Nauk SSSR, Ser. Mat., 32 (1968), 1123-1137 (in Russian).

[4] M. T. KARAEV, On some applications of the ordinary and extended Duhamel products, Sibirskii Matem. Zhurnal, 46, 3( 2005), 553-566 (in Russian).

[5] M. GüRDAL, Description of extended eigenvalues and extended eigenvectors of integration operator on the Wiener algebra, Expo. Math., 27, 2 (2009), 153-160.

[6] M. T. KaraEv, Addition of spectral multiplicities and invariant subspaces, Ph. D. Thesis, Baku, 1991 (in Russian).

[7] M. T. KARAEV, An addition theorem on multiplicities of spectrum, Trudy IMM AN Azerb., 8, 16 (1998), 123-128 (in Russian).

[8] A. SHIELDS, Weighted shift operators and analytic function theory. Topics in operator theory, 128, Math. Surveys, No:13, Amer. Math. Soc., Providence, R.I., 1974, pp. 49.

[9] V. M. KADETS, Bases with individual brackets and bases with individual permutations (Russian), Teor. Funkt. i Funktsional Anal. i Prilozhen, 49 (1988) , 43-51; Translation in J. Soviet. Math., 49 (1990), 1064-1069.

[10] N. K. NiKOLSKI, Selected problems of weighted approximation and spectral analysis, Translated from Proceedings of the Steklov Institute of Mathematics, 120 (1974), 1-270; American Math. Soc., Providence, R.I., 1976, 276pp.

[11] L. V. Kantorovich and G. P. Akilov, Functional Analysis, Nauka, Moscow, 1977.

[12] A. Biswas, A. LAmbert And S. Petrovic, Extended eigenvalues and the Volterra operator, Glasgow Math. J., 44 (2002), 521-534.

[13] A. Biswas, A. Lambert And S. Petrovic, On extended eigenvectors for operators, Preprint.

[14] A. Lambert, Hyperinvariant subspaces and extended eigenvalues, New York J. Math., 10 (2004), $83-88$. 
[15] A. Lambert And S. Petrovic, Beyond hyperinvariance for compact operators, J. Funct. Anal., 219 (2005), 93-108.

[16] A. Biswas, S. Petrovic, On extended eigenvalues of operators, Integral Equations and Operator Theory, 55 (2006), 233-248.

[17] S. PeTrovic, On the extended eigenvalues of some Volterra operators, Integral Equations and Operator Theory, 57 (2007), 593-598.

[18] S. ShKARIN, Compact operators without extended eigenvalues, J. Math. Anal. Appl., 332 (2007), $455-462$. 